\title{
Cosmological birefringence due to CPT-even Chern-Simons-like term with Kalb-Ramond and scalar fields
}

\author{
Shih-Hao Ho ${ }^{1}$, W. F. Kao ${ }^{1, a}$, Kazuharu Bamba ${ }^{2,3, b}$, C. Q. Geng ${ }^{3, c}$ \\ ${ }^{1}$ Institute of Physics, National Chiao Tung University, Hsinchu 300, Taiwan \\ 2 Division of Human Support System, Faculty of Symbiotic Systems Science, Fukushima University, \\ Fukushima 960-1296, Japan \\ ${ }^{3}$ Department of Physics, National Tsing Hua University, Hsinchu 300, Taiwan
}

Received: 4 December 2014 / Accepted: 21 April 2015 / Published online: 5 May 2015

(C) The Author(s) 2015. This article is published with open access at Springerlink.com

\begin{abstract}
We study the CPT-even dimension-six ChernSimons-like term by including dynamical Kalb-Ramond and scalar fields to examine the cosmological birefringence. We show that the combined effect of a neutrino current and a Kalb-Ramond field could induce a sizable rotation polarization angle in the cosmic microwave background radiation polarization.
\end{abstract}

\section{Introduction}

The Lorentz and CPT invariance are foundations of particle physics. Testing the validity of these two invariance principles has been a topic of the highest significance in the field. One of the tests is to use cosmological birefringence [1,2], which is an additional rotation of synchrotron radiation from the distant radio galaxies and quasars. Since it is wavelengthindependent, it is different from Faraday rotation. The first indication of cosmological birefringence was claimed by Nodland and Ralston [3]. Unfortunately, it has been shown that there is no statistically significant signal [4-8]. Nevertheless, this provides a new way to search for new physics in cosmology. In recent years, there are many groups using combined data to constrain this small violation effect. In particular, the analysis by Feng et al. [2] gives $\Delta \alpha=-6.0 \pm 4.0^{\circ}$, while the Wilkinson Microwave Anisotropy Probe (WMAP) group $\Delta \alpha=-1.7 \pm 2.1^{\circ}$ with 5 year data [9]. In addition, the Combined WMAP 5 year data with the BOOMERanG data leads to $\Delta \alpha=-2.6 \pm 1.9^{\circ}[10-13]$, and an improved

\footnotetext{
a e-mail: gore@mail.nctu.edu.tw

b e-mail: bamba@sss.fukushima-u.ac.jp

c e-mail: geng@phys.nthu.edu.tw
}

result by the QUaD Collaboration is $\Delta \alpha=0.64 \pm 0.5 \pm$ $0.5^{\circ}[14,15]$. Combined QUaD, WMAP7, B03 and BICEP data indicates $\Delta \alpha=-0.04 \pm 0.35^{\circ}$ [16]. In a recent paper, the constraint $\Delta \alpha=-0.8 \pm 2.2^{\circ}$ has been reported [17]. It has been pointed out that the Planck Surveyor [18] will reach a sensitivity of $\Delta \alpha$ at levels of $10^{-2}-10^{-3}$ [19], while a dedicated future experiment on the cosmic microwave background radiation polarization would reach $10^{-5}-10^{-6} \Delta \alpha$ sensitivity [19].

It is well known that this phenomenon can be used to test the Einstein equivalence principle as was first pointed out by $\mathrm{Ni}[20,21]$. Another theoretical origin of the birefringence was developed by Carroll et al. [1,4]. They modified the Maxwell Lagrangian by adding a CPT violating Chern-Simons term [1], which results in numerous subsequent works [22-44]. In Ref. [45], a CPT-even dimension-six Chern-Simons-like term was considered, in which the fourvector $p_{\mu}$ is related to a neutrino current [45] and there is a Kalb-Ramond field as an auxiliary field to maintain general gauge invariance. It is clear that the observation of the cosmological birefringence may not imply CPT violation $[46,47]$ but parity violation.

In this paper, we extend the study in Ref. [45] by considering the dynamics of a Kalb-Ramond field and a scalar field. We consider the flat Friedmann-Lemaittre-RobertsonWalker (FLRW) space-time with the metric: $\mathrm{d} s^{2}=-\mathrm{d} t^{2}+$ $a^{2}(t) \mathrm{d} x^{2}$, where $a(t)$ is the scale factor. We use the signature convention for the metric tensor $g=\operatorname{diag}(-,+,+,+)$ and $\epsilon^{\mu \nu \alpha \beta}=(1 / \sqrt{g}) \mathrm{e}^{\mu \nu \alpha \beta}$, where $\mathrm{e}^{\mu \nu \alpha \beta}$ is the Levi-Civita tensor normalized by $\mathrm{e}^{0123}=+1$. We also use units of $k_{\mathrm{B}}=c=\hbar=1$.

The paper is organized as follows. In Sect. 2, we explain the model and derive the equations of motion. We explore the cosmological birefringence in Sect. 3. Finally, conclusions are given in Sect. 4. 


\section{The model}

We start with the string-inspired action [48]

$$
\begin{aligned}
S_{0}= & \int \mathrm{d}^{4} x \sqrt{g}\left[-\frac{1}{2} \epsilon \phi^{2} R-\frac{1}{2} g^{\mu \nu} \partial_{\mu} \phi \partial_{\nu} \phi-V(\phi)\right. \\
& -\frac{\xi_{1}}{6 \phi^{2}} H_{\mu \nu \alpha} H^{\mu \nu \alpha}+\frac{\xi_{2}}{\phi^{2}} j_{\mu}\left(A_{\nu} \tilde{F}^{\mu \nu}+\frac{1}{2} \epsilon^{\mu \nu \alpha \beta} \partial_{\nu} B_{\alpha \beta}\right) \\
& \left.-\frac{1}{4} F^{\mu \nu} F_{\mu \nu}\right],
\end{aligned}
$$

where $\phi$ is the scalar field with the potential $V(\phi), j_{\mu}=$ $\bar{f} \gamma_{\mu} f \equiv\left(j^{0}, \boldsymbol{j}\right)$ is the fermion current, $H_{\mu \nu \alpha} \equiv \partial_{[\mu} B_{\nu \alpha]}$ is the Kalb-Ramond field strength, $F_{\mu \nu}=\partial_{[\mu} A_{\nu]}$, and $\tilde{F}^{\mu \nu}=$ $(1 / 2) \epsilon^{\mu \nu \alpha \beta} F_{\alpha \beta}$ with the electromagnetic vector field $A_{\mu}$; the parameters $\epsilon, \xi_{1}$, and $\xi_{2}$ are unknown constants.

Note that the cosmological birefringence due to a KalbRamond field has been studied in Refs. [49,50]. The major difference with our model is the introduction of a complete fermion current with a nontrivial gauge coupling. Indeed, it is well known that Eq. (1) is not gauge invariant under a gauge transformation because of the interaction $\frac{\xi_{2}}{\phi^{2}} j_{\mu}\left(A_{\nu} \tilde{F}^{\mu \nu}+\right.$ $\left.\frac{1}{2} \epsilon^{\mu \nu \alpha \beta} \partial_{\nu} B_{\alpha \beta}\right)$. We note that, under the gauge transformation $A_{\mu} \longrightarrow A_{\mu}+\partial_{\mu} \theta$, one obtains

$$
\begin{gathered}
\frac{\xi_{2}}{\phi^{2}} j_{\mu}\left(A_{\nu} \tilde{F}^{\mu \nu}+\frac{1}{2} \epsilon^{\mu \nu \alpha \beta} \partial_{\nu} B_{\alpha \beta}\right) \\
\longrightarrow \frac{\xi_{2}}{\phi^{2}} j_{\mu}\left(A_{\nu} \tilde{F}^{\mu \nu}+\frac{1}{2} \epsilon^{\mu \nu \alpha \beta} \partial_{\nu} B_{\alpha \beta}\right) \\
+\frac{1}{2} j_{\mu} \epsilon^{\mu \nu \alpha \beta}\left[\left(\partial_{\nu} \theta\right) F_{\alpha \beta}+\partial_{\nu} \delta B_{\alpha \beta}\right] .
\end{gathered}
$$

The extra term in Eq. (2) from the gauge transformation should be zero, i.e.,

$$
\begin{aligned}
& \frac{1}{2} j_{\mu} \epsilon^{\mu \nu \alpha \beta}\left[\left(\partial_{\nu} \theta\right) F_{\alpha \beta}+\partial_{\nu} \delta B_{\alpha \beta}\right] \\
& \quad=\frac{1}{2} j_{\mu} \epsilon^{\mu \nu \alpha \beta}\left[\partial_{\nu}(\theta) F_{\alpha \beta}+\partial_{\nu} \delta B_{\alpha \beta}\right]=0,
\end{aligned}
$$

which leads to $\delta B_{\alpha \beta}=-\theta F_{\alpha \beta}$. Therefore, we have to modify the field strength tensor of $B_{\mu \nu}$ as

$\tilde{H}_{\mu \nu \alpha} \equiv H_{\mu \nu \alpha}+A_{[\mu} F_{\nu \alpha]}$

As a consequence, the gauge invariant action becomes

$$
\begin{aligned}
S_{0}= & \int \mathrm{d}^{4} x \sqrt{g}\left[-\frac{1}{2} \epsilon \phi^{2} R-\frac{1}{2} g^{\mu \nu} \partial_{\mu} \phi \partial_{\nu} \phi-V(\phi)\right. \\
& -\frac{\xi_{1}}{6 \phi^{2}} \tilde{H}_{\mu \nu \alpha} \tilde{H}^{\mu \nu \alpha}-\frac{1}{4} F^{\mu \nu} F_{\mu \nu} \\
& \left.+\frac{\xi_{2}}{\phi^{2}} j_{\mu}\left(A_{\nu} \tilde{F}^{\mu \nu}+\frac{1}{2} \epsilon^{\mu \nu \alpha \beta} \partial_{\nu} B_{\alpha \beta}\right)\right] .
\end{aligned}
$$

Note that the scalar-tensor coupling considered in this paper was in fact initiated by Brans and Dicke [51-55], motivated by Mach's principle. The Brans-Dicke theory relates the gravitational constant to a dynamical scalar field. The idea that the gravitational constant is a dynamical variable is also coherent with the large number hypothesis proposed by Dirac [56]. Dirac found that a simple combination of some universal constants is close to the age of the universe. It was hence conjectured by Dirac that these constants could be dynamical fields. This also agrees with the original idea of the Weyl invariant theory [57,58]. All coupling constants in a Weyl invariant theory have to be dimensionless if local or global scale invariance is to be preserved. Therefore, in a Weyl invariant theory all dimensionful constants are replaced by dynamical fields with proper order to account for the dimensions of various constants. A dimension-one scalar field is a popular candidate for this purpose. The dimension of a "constant" then emerges as a symmetry breaking effect. Our model is therefore based on the original idea of Weyl, Dirac, and Brans-Dicke.

When a system is in its high energy phase, scale symmetry is preserved. A spontaneous symmetry breaking (SSB) potential is induced in the low energy phase to account for the scale symmetry breaking. The masses of various fields as well as the dimensions of various constants are thus induced when the system grows into its low energy phase. There are a number of ways to introduce a symmetry breaking potential. One simple method is to introduce a $\phi^{4}$ SSB potential of the form $V(\phi)=\lambda\left(\phi^{2}-\phi_{0}^{2}\right)^{2}+V_{0}$ with $\lambda$ a dimensionless constant.

When the system is in its low energy phase, the scalar field will roll down to the local minimum at $\phi=\phi_{0}$. This SSB potential is parity even (it has $\phi \rightarrow-\phi$ symmetry) and hence has been a popular candidate for the purpose of introducing the proper dimension of a parity conserving system. This potential will, however, be plagued by the domain wall problem [59], which requires some more efforts to be settled. We can instead consider a parity odd potential like $V=\lambda\left(\phi-\phi_{0}\right)^{4}+V_{0}$ that is free of the domain wall complex.

Alternatively, one of the other most popular candidates is the Coleman-Weinberg potential of the form that is in agreement with the WMAP observation: $V(\phi)=\frac{\lambda}{4} \phi_{0}^{4}+$ $\lambda \phi^{4}\left[\ln \left(\frac{\phi}{\phi_{0}}\right)-\frac{1}{4}\right]+V_{0}$, with $\phi_{0}$ denoting the vacuum expectation value (VEV) of $\phi$ at the minimum $\phi_{0}$. Note that $V\left(\phi=\phi_{0}\right)=V_{0}$, and the vacuum energy density at the origin is given by $V_{0}+\lambda \phi_{0}^{4} / 4$ [60-64]. The Coleman-Weinberglike potential takes the radiative correction as the origin of SSB. This theory is thus backed up by a convincing dynamical motivation. Note that the form of the potential is not essential in the prediction when the system has settled down to the low energy phase when the scalar field loses its dynamics and sits still at its VEV.

By varying the action with respect to $\phi, g_{\mu \nu}, B_{\mu \nu}$, and $A_{\mu}$, we can have a set of equations of motion as follows: 


$$
\begin{aligned}
& \epsilon \phi R=D_{\mu} \partial^{\mu} \phi-\frac{\partial V}{\partial \phi}+\frac{\xi_{1}}{3 \phi^{3}} \tilde{H}^{2} \\
& -2 \frac{\xi_{2}}{\phi^{3}} j_{\mu}\left(A_{\nu} \tilde{F}^{\mu \nu}+\frac{1}{2} \epsilon^{\mu \nu \alpha \beta} \partial_{\nu} B_{\alpha \beta}\right), \\
& \epsilon \phi^{2} G_{\mu \nu}=\left[\frac{1}{2}\left(\partial_{\alpha} \phi\right)^{2}+V(\phi)\right] g_{\mu \nu}-\partial_{\mu} \phi \partial_{\nu} \phi+\frac{\xi_{1}}{6 \phi^{2}} \tilde{H}^{2} g_{\mu \nu} \\
& +\left(\frac{1}{4} F^{2} g_{\mu \nu}-F_{\mu \alpha} F_{v}^{\alpha}\right)-\frac{1}{\phi^{2}} \tilde{H}_{\mu \alpha \beta} \tilde{H}_{v}^{\alpha \beta} \\
& +\epsilon\left(D_{\nu} D_{\mu} \phi^{2}-D^{\sigma} D_{\sigma} \phi^{2} g_{\mu \nu}\right) \text {, } \\
& D_{\mu}\left(\frac{\xi_{1}}{\phi^{2}} \tilde{H}^{\mu \nu \alpha}+\frac{\xi_{2}}{2 \phi^{2}} \epsilon^{\mu \nu \alpha \beta} j_{\beta}\right)=0, \\
& D_{v} F^{v \mu}-D_{v}\left(\frac{2 \xi_{1}}{\phi^{2}} \tilde{H}^{\nu \alpha \mu} A_{\alpha}+\frac{\xi_{2}}{\phi^{2}} \epsilon^{\beta \alpha \nu \mu} j_{\beta} A_{\alpha}\right) \\
& =\frac{\xi_{1}}{\phi^{2}} \tilde{H}^{\mu \nu \alpha} F_{\nu \alpha}-\frac{\xi_{2}}{\phi^{2}} j_{\nu} \tilde{F}^{\nu \mu} .
\end{aligned}
$$

Since $\tilde{H}^{\mu \nu \alpha}$ is a totally antisymmetric tensor, we can write $\tilde{H}^{\mu \nu \alpha}=\epsilon^{\mu \nu \alpha \beta} T_{\beta}$, where $T_{\beta}$ is a vector with mass dimension three.

Thus, Eq. (8) is rewritten

$\epsilon^{\mu \nu \alpha \beta} \partial_{\mu}\left(\frac{\xi_{1}}{\phi^{2}} T_{\beta}+\frac{\xi_{2}}{2 \phi^{2}} j_{\beta}\right)=0$.

Focusing on the space-time manifold with first trivial homology group, any closed one-form is an exact one-form. Therefore, from Eq. (10), we can express the torsion field as

$\frac{1}{\phi^{2}}\left(\xi_{1} T_{\beta}+\frac{\xi_{2}}{2} j_{\beta}\right)=\partial_{\beta} \Phi$,

where $\Phi$ is a dimensionless pseudo-scalar. With the help of Eq. (11), we can further simplify the equations of motion to

$$
\begin{aligned}
& \epsilon \phi R=D_{\mu} \partial^{\mu} \phi-\frac{\partial V}{\partial \phi}-\frac{2 \phi}{3 \xi_{1}}\left(\partial_{\mu} \Phi\right)^{2}+\frac{\xi_{2}^{2}}{2 \xi_{1} \phi^{3}}\left(j_{\mu}\right)^{2}, \\
& \epsilon \phi^{2} G_{\mu \nu}= {\left[\frac{1}{2}\left(\partial_{\alpha} \phi\right)^{2}+V(\phi)\right] g_{\mu \nu} } \\
&-\partial_{\mu} \phi \partial_{\nu} \phi+\epsilon\left(D_{\nu} D_{\mu} \phi^{2}-D^{\sigma} D_{\sigma} \phi^{2} g_{\mu \nu}\right) \\
&+\frac{1}{\xi_{1} \phi^{2}}\left[\phi^{4}\left(\partial_{\alpha} \Phi\right)^{2}-\xi_{2} \phi^{2} j_{\alpha} \partial^{\alpha} \Phi+\frac{\xi_{2}^{2}}{4}\left(j_{\alpha}\right)^{2}\right] g_{\mu \nu} \\
&-2 \frac{\xi_{1}}{\phi^{2}}\left(\frac{\phi^{2}}{\xi_{1}} \partial_{\mu} \Phi-\frac{\xi_{2}}{2 \xi_{1}} j_{\mu}\right)\left(\frac{\phi^{2}}{\xi_{1}} \partial_{\nu} \Phi-\frac{\xi_{2}}{2 \xi_{1}} j_{\nu}\right) \\
&+\left(\frac{1}{4} F^{2} g_{\mu \nu}-F_{\mu \alpha} F_{v}^{\alpha}\right), \\
& D_{\mu} F^{\mu \nu}=-4\left(\partial_{\mu} \Phi\right) \tilde{F}^{\mu \nu} .
\end{aligned}
$$

\section{Cosmological birefringence}

For the present purpose, we need not specify the form of the potential for the scalar field. Therefore, we simply consider the potential to be the cosmological constant $V_{0}$ when the scalar field is at its VEV $\phi_{0}$. Taking all the $\phi$ fields in the equations to be $\phi_{0}$, the equations of motion become

$$
\begin{aligned}
\epsilon \phi_{0} R=-2 & \frac{\phi_{0}}{\xi_{1}}\left(\partial_{\mu} \Phi\right)^{2}+\frac{\xi_{2}^{2}}{2 \xi_{1} \phi_{0}^{3}}\left(j_{\mu}\right)^{2} \\
\epsilon \phi_{0}^{2} G_{\mu \nu}= & V_{0} g_{\mu \nu}+\left(\frac{1}{4} F^{2} g_{\mu \nu}-F_{\mu \alpha} F_{\nu}^{\alpha}\right) \\
& +\frac{1}{\xi_{1} \phi_{0}^{2}}\left[\phi_{0}^{4}\left(\partial_{\alpha} \Phi\right)^{2}-\xi_{2} \phi_{0}^{2} j_{\alpha} \partial^{\alpha} \Phi+\frac{\xi_{2}^{2}}{4}\left(j_{\alpha}\right)^{2}\right] g_{\mu \nu} \\
& -2 \frac{\xi_{1}}{\phi_{0}^{2}}\left(\frac{\phi_{0}^{2}}{\xi_{1}} \partial_{\mu} \Phi-\frac{\xi_{2}}{2 \xi_{1}} j_{\mu}\right)\left(\frac{\phi_{0}^{2}}{\xi_{1}} \partial_{\nu} \Phi-\frac{\xi_{2}}{2 \xi_{1}} j_{\nu}\right) .
\end{aligned}
$$

Taking the trace of Eq. (16), we have

$$
\begin{aligned}
-\epsilon \phi_{0}^{2} R= & 4 V_{0}+2 \frac{\phi_{0}^{2}}{\xi_{1}}\left(\partial_{\mu} \Phi\right)^{2}-2 \frac{\xi_{2}}{\xi_{1}} j_{\mu} \partial^{\mu} \Phi \\
& +\frac{\xi_{2}^{2}}{2 \xi_{1} \phi_{0}^{2}}\left(j_{\mu}\right)^{2} .
\end{aligned}
$$

By combining Eqs. (15) and (17), we obtain

$$
4 V_{0}-2 \frac{\xi_{2}}{\xi_{1}} j_{\mu}\left(\partial^{\mu} \Phi\right)+\frac{\xi_{2}^{2}}{\xi_{1} \phi_{0}^{2}}\left(j_{\mu}\right)^{2}=0 .
$$

In the FLRW universe, it is reasonable to assume a homogeneous and isotropic fermion current and torsion field [45], i.e., $j_{\mu}=\left(j_{0}(t), \mathbf{0}\right)$ and $T_{\mu}=\left(T_{0}(t), \mathbf{0}\right)$. From Eq. (18), we have the evolution equation for the dimensionless pseudoscalar $\Phi$ :

$4 V_{0}+2 \frac{\xi_{2}}{\xi_{1}} j_{0}\left(\partial_{0} \Phi\right)-\frac{\xi_{2}^{2}}{\xi_{1} \phi_{0}^{2}}\left(j_{0}\right)^{2}=0$.

The solution of Eq. (19) can easily be derived as

$\partial_{0} \Phi=-2 \frac{\xi_{1} V_{0}}{\xi_{2} j_{0}}+\frac{\xi_{2}}{2 \phi_{0}^{2}} j_{0}$.

Similar to the calculation in Ref. [45], the change in the position angle of the polarization plane $\Delta \alpha$ at the redshift $z \equiv 1 / a-1$ is given by

$$
\begin{aligned}
\Delta \alpha & =2 \int\left(\partial_{0} \Phi\right) \frac{\mathrm{d} t}{a(t)} \\
& =2 \int_{0}^{1100}\left(-2 \frac{\xi_{1} V_{0}}{\xi_{2} j_{0}}+\frac{\xi_{2}}{2 \phi_{0}^{2}} j_{0}\right) \frac{\mathrm{d} z}{H_{0}(1+z)^{n}}
\end{aligned}
$$

where $H_{0}=2.1 \times 10^{-42} h \mathrm{GeV}$ is the Hubble constant with $h \simeq 0.7$ at present and we have assumed our universe is flat and $n=3 / 2,2,0$ correspond to a matter-, radiation-, and vacuum-dominated universe, respectively. To estimate $\Delta \alpha$ in Eq. (21), we take the zero component of the fermion current $j_{0}$ to be the (lightest) neutrino asymmetry, say, the electron neutrino in our universe, 


$$
\begin{aligned}
j_{0} & =\Delta n_{v_{e}}=\frac{1}{12 \zeta(3)}\left(\frac{T_{v_{e}}}{T_{\gamma}}\right)^{3} \pi^{2} \xi_{v_{e}} n_{\gamma} \\
& =\frac{2}{33} \xi_{v_{e}} T_{\gamma_{0}}^{3}(1+z)^{3},
\end{aligned}
$$

where $T_{\gamma_{0}}$ is the CMB temperature at present, $\xi_{v_{e}}$ is the degeneracy parameter for the electron neutrino, and $\left(T_{v_{e}} / T_{\gamma}\right)^{3}=$ $4 / 11$ is assumed. In Ref. $[65,66]$ the bound on the degeneracy parameter is $-0.046<\xi_{v_{e}}<0.072$ for a $2 \sigma$ range of the baryon asymmetry.

Inserting Eq. (22) into Eq. (21), we have

$\Delta \alpha=\left.2 f(z)\right|_{0} ^{1100}$

where $f(z)$ is given by

$$
\begin{aligned}
f(z)= & \left(\frac{33 \xi_{1} V_{0}}{(n+2) \xi_{2} \xi_{v_{e}} T_{\gamma_{0}}^{3} H_{0}}\right)(1+z)^{-(n+2)} \\
& +\left(\frac{\xi_{2} \xi_{v_{e}} T_{\gamma_{0}}^{3}}{33(n-4) \phi_{0}^{2} H_{0}}\right)(1+z)^{(4-n)} .
\end{aligned}
$$

Therefore, there is a bound on the function $|f(z)|$

$$
|f(z)| \geq 2\left[\frac{\xi_{1} V_{0}}{(n+2)(n-4) \phi_{0}^{2} H_{0}^{2}(1+z)^{2(n-1)}}\right]^{1 / 2},
$$

which can be thought of as a bound on the contribution of the effective cosmological constant $V_{0}$. The parameters in our model have constraints from observations, which gives us $\left|\frac{\xi_{1} V_{0}}{\xi_{2}}\right| \leq 10^{-85} \mathrm{GeV}^{4}$ and $\left|\frac{\xi_{2}}{\phi_{0}^{2}}\right| \leq 10^{-8} \mathrm{GeV}^{-2}$; $\left|\frac{\xi_{1} V_{0}}{\xi_{2}}\right| \leq 10^{-85} \mathrm{GeV}^{4}$ and $\left|\frac{\xi_{2}}{\phi_{0}^{2}}\right| \leq 10^{-7} \mathrm{GeV}^{-2}$, and $\left|\frac{\xi_{1} V_{0}}{\xi_{2}}\right| \leq 10^{-86} \mathrm{GeV}^{4}$ and $\left|\frac{\xi_{2}}{\phi_{0}^{2}}\right| \leq 10^{-13} \mathrm{GeV}^{-2}$ for a matter-, radiation-, and vacuum-dominated universe. Note that the effective coupling constant $\phi_{0}^{2}$ is constrained to be the reduced Planck mass $M_{\mathrm{pl}}^{2}=1 / 8 \pi G$ because of the EinsteinHilbert action. By taking $\epsilon=1, V_{0} \sim 10^{-85}(\mathrm{GeV})^{4}, \xi_{1}=1$, $\xi_{2}=1, \xi_{v_{e}} \sim 10^{-3}$, and $n=3 / 2$ for a matter-dominated universe, we get $\Delta \alpha \sim-9.7 \times 10^{-2}$, which could explain the results in Refs. [2,9-15].

\section{Conclusions}

In the present paper, we have studied the CPT-even dimension-six Chern-Simons-like term in Ref. [45] by including dynamical torsion and scalar fields to explain the cosmological birefringence effect. The combined effect of the Kalb-Ramond field and the neutrino current induces a sizable rotation polarization angle in the CMB data provided that there is a non-zero neutrino number asymmetry.

It is interesting to note that the effect induced by the KalbRamond field is the inverse of the one due to the neutrino current, as shown in Eq. (24). In contrast to the model in
Ref. [45], in which a similar dimension-six interaction with an undetermined effective coupling constant was examined, we consider, however, the dynamical scalar fields in terms of the coupling constants of the Ricci scalar, the Kalb-Ramond field, and in interaction terms. Namely, the effective coupling constant $\phi_{0}^{2}$ is related to $M_{\mathrm{pl}}$ in the Einstein-Hilbert action. Because of this limitation, the contribution to the angle $\Delta \alpha$ is highly suppressed to $O\left(10^{-32}\right)$, and the corresponding $V_{0}$ has to be around $10^{-85}(\mathrm{GeV})^{4}$ to match the current observational constraint.

Finally, we remark that there should be other interesting cosmological phenomena in this model [67], which will be studied elsewhere.

Acknowledgments The work by S.H.H. and W.F.K. is supported in part by the Ministry of Science and Technology (MOST) of Taiwan under Contract No. NSC-98-2112-M-009-002-MY3 and NSC 1012112-M-009-004-MY3. C.Q.G. is supported in part by the National Science Council of R.O.C. under Grant No. NSC-101-2112-M-007-006MY3 and National Tsing Hua University under Grant No. 104N2724E1. K.B. is supported in part by the JSPS Grant-in-Aid for Young Scientists (B) No. 25800136 (K.B.) and National Tsing Hua University under the Boost Program and Grant No. 99N2539E1.

Open Access This article is distributed under the terms of the Creative Commons Attribution 4.0 International License (http://creativecomm ons.org/licenses/by/4.0/), which permits unrestricted use, distribution, and reproduction in any medium, provided you give appropriate credit to the original author(s) and the source, provide a link to the Creative Commons license, and indicate if changes were made.

Funded by SCOAP ${ }^{3}$.

\section{References}

1. S.M. Carroll, G.B. Field, R. Jackiw, Phys. Rev. D 41, 1231 (1990)

2. B. Feng, M. Li, J.Q. Xia, X. Chen, X. Zhang, Phys. Rev. Lett. 96, 221302 (2006)

3. B. Nodland, J.P. Ralston, Phys. Rev. Lett. 78, 3043 (1997)

4. S.M. Carroll, G.B. Field, Phys. Rev. Lett. 79, 2394 (1997)

5. D.J. Eisenstein, E.F. Bunn, Phys. Rev. Lett. 79, 1957 (1997)

6. B. Nodland, J.P. Ralston, Phys. Rev. Lett. 79, 1958 (1997)

7. J.P. Leahy, arXiv:astro-ph/9704285

8. B. Nodland, J.P. Ralston, arXiv:astro-ph/9706126

9. E. Komatsu et al., WMAP Collaboration, Astrophys. J. Suppl. 180, 330 (2009)

10. T.E. Montroy et al., Astrophys. J. 647, 813 (2006)

11. W.C. Jones et al., Astrophys. J. 647, 823 (2006)

12. F. Piacentini et al., Astrophys. J. 647, 833 (2006) (see also http:// cmb.phys.cwru.edu/boomerang/)

13. J.Q. Xia, H. Li, G.B. Zhao, X. Zhang, Astrophys. J. 679, L61 (2008)

14. E.Y.S. Wu et al., QUaD Collaboration, Phys. Rev. Lett. 102, 161302 (2009). arXiv:0811.0618 [astro-ph]

15. M.L. Brown et al., QUaD collaboration, Astrophys. J. 705, 978 (2009)

16. J.Q. Xia, H. Li, X. Zhang, Phys. Lett. B 687, 129 (2010)

17. S.d.S. Alighieri, F. Finelli, M. Galaverni, Astrophys. J. 715, 33 (2010) arXiv:1003.4823 [astro-ph.CO]

18. The Planck Consortia, The scientific Case of Planck, ESA Publication Division (2005) (see also http://www.rssd.esa.int/index.php? project=PLANCK) 
19. W.T. Ni, Chin. Phys. Lett. 22, 33 (2005)

20. W.T. Ni, Phys. Rev. Lett. 38, 301 (1977)

21. W.T.Ni, Int. J. Mod. Phys. D 14, 901 (2005) (see for a recent review)

22. A. Lue, L.M. Wang, M. Kamionkowski, Phys. Rev. Lett. 83, 1506 (1999). arXiv:astro-ph/9812088

23. B. Feng, H. Li, M. Li, X. Zhang. Phys. Lett. B 620, 27 (2005). arXiv:hep-ph/0406269

24. J. Alfaro, A.A. Andrianov, M. Cambiaso, P. Giacconi, R. Soldati, Phys. Lett. B 639, 586 (2006). arXiv:hep-th/0604164

25. G.C. Liu, S. Lee, K.W. Ng, Phys. Rev. Lett. 97, 161303 (2006). arXiv:astro-ph/0606248

26. T. Kahniashvili, G. Gogoberidze, B. Ratra, Phys. Lett. B 643, 81 (2006). arXiv:astro-ph/0607055

27. D. O'Dea, A. Challinor, B.R. Johnson, Mon. Not. R. Astron. Soc. 376, 1767 (2007). arXiv:astro-ph/0610361

28. A.J. Hariton, R. Lehnert, Phys. Lett. A 367, 11 (2007). arXiv:hep-th/0612167

29. V.A. Kostelecky, M. Mewes, Phys. Rev. Lett. 99, 011601 (2007). arXiv:astro-ph/0702379

30. P. Cabella, P. Natoli, J. Silk, Phys. Rev. D 76, 123014 (2007). arXiv:0705.0810 [astro-ph]

31. F. Finelli, M. Galaverni, Phys. Rev. D 79, 063002 (2009). arXiv:0802.4210 [astro-ph]

32. T. Kahniashvili, R. Durrer, Y. Maravin, Phys. Rev. D 78, 123009 (2008). arXiv:0807.2593 [astro-ph]

33. M. Pospelov, A. Ritz, C. Skordis, A. Ritz, C. Skordis, Phys. Rev. Lett. 103, 051302 (2009). arXiv:0808.0673 [astro-ph]

34. V.A. Kostelecky, M. Mewes, Astrophys. J. 689, L1 (2008). arXiv:0809.2846 [astro-ph]

35. F.A. Brito, L.S. Grigorio, M.S. Guimaraes, E. Passos, C. Wotzasek, Phys. Rev. D 78, 125023 (2008). arXiv:0810.3180 [hep-th]

36. M. Li, X. Zhang, Phys. Rev. D 78, 103516 (2008). arXiv:0810.0403 [astro-ph]

37. J.Q. Xia, H. Li, G.B. Zhao, X. Zhang, Int. J. Mod. Phys. D 17, 2025 (2008). arXiv:0708.1111 [astro-ph]

38. J.Q. Xia, H. Li, X.1. Wang, X. Zhang, Astron. Astrophys. 483, 715 (2008). arXiv:0710.3325 [hep-ph]

39. D. Maity, S. SenGupta, S. Sur, Phys. Rev. D 72, 066012 (2005). arXiv:hep-th/0507210 (background)

40. D. Maity, P. Majumdar, S. SenGupta, JCAP 0406, 005 (2004). arXiv:hep-th/0401218 (anisotropy)

41. D. Maity, S. SenGupta, S. Sur, Eur. Phys. J. C 42, 453 (2005). arXiv:hep-th/0409143
42. D. Maity, S. SenGupta, Class. Quant. Grav. 21, 3379 (2004). arXiv:hep-th/0311142 (with torsion)

43. S. SenGupta, S. Sur, JCAP 0312, 001 (2003). arXiv:hep-th/0207065

44. S. Kar, P. Majumdar, S. SenGupta, S. Sur, Class. Quant. Grav. 19, 677 (2002). arXiv:hep-th/0109135

45. C.Q. Geng, S.H. Ho, J.N. Ng, JCAP 0709, 010 (2007)

46. C.Q. Geng, S.H. Ho, J.N. Ng, Int. J. Mod. Phys. A 23, 3408 (2008)

47. C.Q. Geng, S.H. Ho, J.N. Ng, Can. J. Phys. 86, 587 (2008)

48. M. Green, J. Schwarz, E. Witten, Superstring Theory, Vol II. (Cambridge, 1985)

49. S. Kar, P. Majumdar, S. SenGupta, A. Sinha, Eur. Phys. J. C 23, 357 (2002). arXiv:gr-qc/0006097

50. K.R.S. Balaji, R.H. Brandenberger, D.A. Easson, JCAP 0312, 008 (2003). arXiv:hep-ph/0310368

51. C. Brans, R.H. Dicke, Phys. Rev. 124, 925 (1961)

52. R.H. Dicke, ibid. 125, 2163 (1962)

53. K. Uehara, C.W. Kim, Phys. Rev. D 26, 2575 (1982)

54. L. Perivolaropoulos, JCAP 10, 001 (2005)

55. B. Boisseau, Phys. Rev. D 83, 043521 (2011)

56. P.A.M. Dirac, Cosmological models and the large numbers hypothesis. Proc. R. Soc. Lond. A 338(1615), 439446 (1974). doi:10. 1098/rspa.1974.0095

57. H. Weyl, Ann. Phys. 54, 117 (1917)

58. H. Weyl, Ann. Phys. 59, 101 (1919)

59. E.W. Kolb, M.S. Turner, in Frontiers in Physics, vol. 70, p. 719 (Addison-Wesley, Redwood City, 1988)

60. S.R. Coleman, E. Weinberg, Phys. Rev. D 7, 1888 (1973)

61. A. Albrecht, R.H. Brandenberger, Phys. Rev. D 31, 1225 (1985)

62. A. Albrecht, R.H. Brandenberger, R. Matzner, Phys. Rev. D 32, $1280(1985)$

63. A.D. Linde, Contemporary Concepts in Physics, vol. 5, Particle physics and inationary cosmology (Harwood Academic, Chur, 1990)

64. Q. Shafi, V.N. enouz, Phys. Rev. D 73, 127301 (2006)

65. P.D. Serpico, G.G. Raffelt, Phys. Rev. D 71, 127301 (2005)

66. V. Simha, G. Steigman, JCAP 0808, 011 (2008) (see also for an updated discussion)

67. W.F. Kao, Phys. Rev. D 46, 5421 (1992) (see for example) 